\section{THROMBO-ANGIITIS OBLITERANS.}

Sir W. I. de C. WHELLER, F.R.C.S.I., SURGEON, MERCER'S HOSPITAL, DUBLIN.

The name thrombo-angiitis obliterans was suggested in 1908 by Buerger for this interesting and remarkable disease, and has been accepted almost miversally in the United States. 'Two cases have come under my care which illustrate the tragic and mysterious nature of its clinical manifestations.

The literature on the subject is sparse, but those who have made it a special study believe that it almost exclusively occurs in male adult Jews, especially in the poores class of $\mathbf{J e w}$ who has migrated from Russia to America or elsewhere. It has been found also in Central and SoutiEastern Europe, and appears to have been especially prevalent during the war. The disease is almost unknown among the inhabitants of these countries, but it has been studied by Parkes Weber in London, who admirably describes the condition in the Quarterly Journal of Medicine (vol. 9, 1915-16). Most of the original work comes from Leo Buerger of New York. It is sometimes referred to as Buerger's discase.

For no ascertainable reason some inflammatory condition arises in the deep vessels of the extremities, usually the legs, ending in thrombosis and obliteration, as the namo implies. At the onset there may be no clinical evidence of the existence of such a condition, but as time goes on -weeks, months, or years-the organization of the inflammatory products proceeds, until finally local svncope and death may supcrvene. When a point is reached at which the circulation is at a minimum, and a struggle is taking place to maintain the vitality of the part, the pain often becomes unendurable, and sooner or later, in the majority of cases, amputation becomes inevitable, either for the relief of pain or in anticipation of a spreading gangrene. It is to be emphasized that the disease is pre-senile, that it is non-syphilitic, and is not embolic in origin. Frequently the disease in the arteries is accompanied cr followed by thrombosis of the veins. The disease does not progress continuously; surprising changes, both for better and for worse, are the impressive features of the variegated clinical picture of thrombo-angiitis obliterans. The attempts of Nature to provide a new circulation, either by collateral anastomosis or by the canalization of the thrombosed blood vessels and connective tissues, is mentioned by all the writers. From a study of the published cases it would appear that this attempt is usually followed in the end by clinical, as distinct from pathological, failure. Thus the disease has a tendency to periodic relapse, and may end fatally when some important vessel is attacked. The two cases in Dublin which came under our care were diagnosed more by a process of exclusion than by anything tangible or concrete which could be obtained from the history or by clinical examination. Syphilis was first excluded; Raynaud's disease and the other angio-neuroses, together with endarteritis obliterans, were then eliminated. The urine in both cases was sugar-free, and there was no cardiac or other discoverable lesion remoto from the affecte. 1 parts. Buerger admits that only in very few cases can the symptoms justify a diagnosis in the incipient stage; it is only when we compare the history with the subsequent events that we can relegate definite signs to the onset of the vascular changes. After all, it is from the healing of the vascular lesion, with the consequent disastrous occlusive thrombosis, that the diagnosis is made. In both the cases here reported, and in nearly all the other's recorded, pain in the calf of the leg, of the trpe known as intermittent claudication, was an early sign. An angry red colour of the foot, when allowed to hang, together with puffy toes, is a constant sign when the disease is woll established.

Trophic lesions may be the first sign, and there are acute cases in which gangrene very rapidly develops. The initial symptoms may be too slight to attract attention. Both legs may be intensively involved with thrombo-angiitis obliterans, yet one shows outward signs of disease, the other, to all intents and purposes, appearing normal to the patient, but the pulse is absent in the dorsalis pedis, posterior tibial, and popliteal arteries. Cases suffering only from intermittent claudication have many times been treated for rheumatism, for gout, for flat-foot, and other orthopaedic conditions. Thrombo-angiitis obliterans in such cases is neither thought of nor looked for, but if a careful examination is made, in addition to pulsoless vessels, blanching and reactionary redness becomes apparent on changing the position of the foot: A history of thrombo-phlebitis should always be sought. In one case mentioned by Buerger, of a Russian Hebrew, aged 32, all the vessels of both lower extremities, from the femorals down, were pulseless. The redness and the blanching were obvious, but there were no trophic disturbances, the patient complained of no pain, and considered himself perfectly well.

In attempting to classify the discase, Buerger suggests: (1) the typical form in the lower extremities; (2) the same with associated thrombo-phlebitis; (3) the samo with involvement of the upper extremities; (4) a form difficult to diagnose in more scnile patients who suffer from secondary or complicating arterio-sclerosis. I think that any one of these types may be acute, or subacute and intermittent, or chronic and intermittent.

Weber, in his close study of the disease amongst adult Jewish males resident in London, makes some interesting observations. One of his patients was aged only 1 vear, and another only $2 \frac{1}{2}$ years, when they migrated to England from the Fast. He states that the affection occurs almost exclusively among Jews, but that it is not absolutely limited to the poorer classes. In nearly every case there was a history of habitual cigarette smoking (the brand of cigaretto had no significance), and he thinks this is a contributory factor in producing the disease. The essential cause of the disease still remains unknown. Weber emphasizes the fact that acquired or inherited syphilis is almost invariably absent. It will be seen later that the two cases which form the basis of this communication might well have served as illustrations for his remarks.

"The characteristic symptoms of the disease are: (1) redness or cyanosis of the foot when it is allowed to rest in the dependent position; (2) pallor of the foot on movement of the ankle-joint; (3) intermiltent ciaudication when the patient walks for a few minutes; (4) absence of pulsation in the arteries of the foot, most noticeable in the dorsalis pedis artery."

Some confusion has arisen owing to the use of the term "erythromelalgia." It was originally employed by Wcir Mitchell for vague conditions of an extremity associated with pain and redness or cyanosis. It is not a diagnostic term, and might be applied to frostbito as well as disorders of functional origin, or any of the many angio-neurotic conditions. The disease never occurs in a woman. "The Jewish women," says Weber, " in the East End of London are all abstainers from tobacco; their men folk are notoriously fond of it."

\section{Treaturent}

The question of treatment can only be discussed in a general way. Each caso must be judged on its merits. Tho pains may reach an intolerable pitch and call for amputation within a few weeks in acute cases. There is a difference of opinion as to whether amputation should be performed high or low. I am convinced that where there is any doubt high amputation is best. It is the well established surgical procedure for gangrene which is spreading beyond the toes. Knowing the pathology of the discase, the rules which guide us in dealing with senile gangrene may bo reasonably followed. Sir Anthony Bowlby' says that the best course is to wait and see to what extent tho collateral circulation is capable of dealing with the part. When senile gangrene is not limited to the toes, but has spread to the dorsum of the foot, amputation should be carried out without delay. The operation should be performed immediately above the knee. Buerger states that in the majority of cases amputation of at least a portion of the leg will become necessary, and that the Stokes-Gritti operation is the ideal procedure. Low amputations have beon occasionally successful, but the risk of failure is too great, as is illustrated in both the cases mentioned below. 
Conservative Treatment.

Buerger recommends an ingenious method of encouraging the capillary eirculation, a method somewhat analogous to the contrast baths recommended by Sir Robert Jones in orthopaedic conditions. Buerger's plan is to induce reactionary hyperaemia in the affected part by a preliminary elevation of the leg followed by its depression into a dependent position. It is effectual in the milder form of cases if the pain can be tolerated. The period of elevation should be the minimum amount of time necessary to produce a frank blanching of the foot; this takes from thirty seconds to three minutes. The leg is then allowed to hang for one or two minutes beyond the time necessary for the induction of a distinct blush. The third position is that of rest with the leg horizontal. The sittings should be repeated frequently, during the day. Passive hyperaemia by Bier's suction method has been helpful. The apparatus must be large enough to enclose the front part of the foot; the application is made for five to fifteen minutes three times a week. ${ }^{2}$ Ligation of the femoral vein below the entrance of the long saphenous vein has been tried without success. $^{3}$ It is difficult to see on what basis some authorities recommend the intravenous injection of sodium citrate. The anti-coagulating action of sodium citrate in the employment of blood transfusion is followed by a coagulating effect in the case of the recipient's blood, and it is now well known that whether sodium citrate is given intramuscularly, intravenously, or subcutaneously, the result is prompt and pronounced shortening of the coagulating time. It is for this reason that injections of sodium citrate are effective in cases of haemorrhage.

Arterio-renous anastomosis has been, for obvious reasons, unsuccessful, and Leriche's periarterial sympathectomy not only failed in both the cases under review, but in every other case on record in which it was tried. For the relief of thrombo-angiitis obliterans the nueration should now be abandoned. Alcohol has been injected into the posterio: tibial nerve at the ankle for the alleviation of pain with success. The anaesthesia of the sole of the foot and the paralysis of the intrinsic muscles which follows make practically no difference in walking.

The outstanding features in the history of the two following cases were: (1) Neither was a Hebrew; (2) neither had syphilis; (3) both had served abroad; (4) both were heany cigarette smokers; $(5)$ the first patient lost portions of all four limbs during a period of about ten years; (6) the second case was of the acute variety, and the patient lost the left leg above the knee within a few weeks of the first attack of pain in his calf.

Case I.

This patient was not' directly under my care, but I saw him many times during his three years' treatment in Blackrock Hospital. The case illustrates the tragic course which the disease than thirty occasions. The patient served during the war in the navy, at first in the North Sea, and subsequently in the tropics. He remembers that he was off duty for a day or two at a time owing to cramps in his legs. In 1915 the right foot became particularly troublesome; the big toe, he states, became white and lost sensatroublesome; the big toe, he states, became white and lost sensa-
tion. These attacks were transitory. In 1919 he was admitted to tion. These attacks were transitory: In 1919 he was admitted to
the Haulbowline Hospital at Cobh (Queenstown); the great toe of his right foot had become black; it and the neighbouring three toes were removed. He was nine months in this hospital, but the wounds never healed. He was discharged, and remained at home for orie and a half years. When at home the great toe of the other foot (left) became black, and he was admitted to a Dublin hospital, - where a Syme's amputation was performed in 1922 . The stump became gangrenous, and, as in the case of the toes of the other foot, the granulating wounds took eight months to heal. Dther foot, the granulating wounds took eight months to heal.
About a year later he was admitted to Blackrock Hospital with spreading gangrene of the right foot (from which the toes had been removed in 1919), and a modified Lisfranc amputation wa performed. The flaps became gangrenous, and after many local operations amputation was performed below the knee. Necrosis of the bone followed, which necessitated a further operation, and a femoral sympathectomy was attempted at the same time (May, 1924, one year after admission). In 1925 the left leg (from which the foot had been removed in 1922) showed signs of slowly weveloping gangrene, and an amputation was performed below the knee eight months later. Once again the flaps became discoloured and gangrenous, and a month later amputation was performed in the ?ower third of the thigh. Almost simultaneously with the development of the gangrene in the stump of the left leg trouble commenced in the left hand. Blushing and blanching, with excruciating pain in the tips of the fingers, commenced, as in the case of the toes. The tips of the little and ring fingers sloughed off. Three months later the tips of the fingers and thumb of the opposite hand went through a similar but modified process. Mercifully, the spreading process stopped when the tips of the phalanges were protruding through the finger pulps. It became necessary, however, to amputate the first finger of the right hand. His present condition is : Left leg amputated through lower third of thigh; right leg amputated below the knee; right hand, first finger amputated, nails and tips of the other fingers
involved; left hand, nails and tips of fingers also affected-the involved; left hand, nails and tips of fingers also affected-tho middle finger is missing from an accident in childhood. There is
a distinct rubor to be seen on both ulnar and radial sides of both a distinct rubor to be seen on both ulnar and radial sides of both
palms. Both radial pulses are good, both femoral arteries are pulsating below Poupart's ligament.

Raynaud's disease cannot absolutely be excluded in the diagnosis of this case, but against it is the fact that the patient was and is a robust sailor who contracted the disease at the age of 25 . All the preliminary phenomena were confined to the feet. The gangrenous process was deep and destructive in the lower limbs over a period exceeding ten rears. Lewis and Leichert ${ }^{4}$ mention a similar case. They suggest ligature of the femoral artery distal to the profunda as a means of forcing and accelerating the development of collateral circulation through more healthy channels.

Case II.

An officer, aged 28, who had served in Egypt for a number of years, in the middle of August, 1926, was boarding a ship
cn route for home when he had a sudden severe pain in the calf cn route for home when he had a sudden severe pain in the calf
of his left leg. His account of the sudden pain-as if someone had hit him from behind-reminded one of the typical history given when the plantaris tendon is ruptured. On arrival home The condition of the leg was quite characteristic of thromboangiitis obliterans. When the foot was in the dependent position angitis obliterans. When the foot was in the dependent position it was flushed, and had an appearance as if it had been immersed
in very hot water. When the foot was raised to the horizontal position only a few seconds elapsed until it became blanched and marble white. The pain then became excruciating, and could not be borne for more than a minute. When allowed to hang once again the capillary flush rapidly reappeared, and the pain was markedly relieved. The patient slept with the leg hanging out over the side of the bed, supported on a low chair and cushion. The arteries round the foot were pulseless. Nothing could be obtained from the history to throw light on the local condition, obtained from the history to throw light on the local condition, and a thorough routine examination gave equally negative results. In making the diagnosis we were faced with the fact that this half-fed type of patient which usually contracts the disease; in fact, he was a strong, young, athletic officer, fond of polo and pig-sticking, and many other' athletic activities. Embolism was excluded in the absence of cardiac defect and of any recent illness. It was concluded also that if the limb survived the first shock of sudden arterial occlusion from embolus, in three weeks all anxiety about collateral circulation would have disappeared. The anxiety about collateral circulation would have disappeared. The reverse occurred; each few days marked a further diminution in
vascular supply. The diagnosis of pre-senile, non-syphilitic endarteritis obliterans was made, a term which in cryptogenetic cases is interchangeable with thrombo-angiitis obliterans, but not so expressive. We had hoped that, as gangrene had not actually appeared in the first two or three weeks, the collateral circulation would become more and more established in a young, vigorous man, and that the limb would be saved; but, on the contrary, the case was of the acute form; trophic sores developed on the great
toe and the neighbouring toe, lymphangitis followed, and the toe and the neighbouring toe, lymphangitis followed, and the glands became enlarged in the groin. The patient became worn
out with pain, morphine had little or no effect, and amputation out with pain, morphine had little or no effect,

was performed above the knee on September 18th. $x$-rayed to discover if signs of calcification were present; when the femoral artery was exposed high up to perform the Leriche operation, some days prior to amputation, it felt normal in every respect. The stripping of the artery was not attended with any respect. The stripping of the artery was difficulty, such as has been reported by other writers. It unusual difficulty, such as has been reported by of of the artery was observed, however, that primary contraction of the artery
when the sympathetic sheath was in the process of removal did not occur.

During the three weeks at our disposal from the time of the first pain in the calf to the amputation above the knee conservative treatment, in addition to the Leriche operation, was rigorously employed. Hot-air baths, diathermy, immersion of the foot in heated wax, to mention just a few of

Dr. T. T. O'Farrell, who examined the limb after removal, found the popliteal artery blocked by an old thrombus one inch below the level of amputation. Old-standing endarteritis was obvious here and in the vessels supplying the foot. Thrombo-phlebitis, with occlusion and calcification of the deep veins, was also found. Canalization of the thrombi and of the connective tissues completed the pathological picture.

\section{REFERENCES.}

Lancet, 1924, ii, 1122. 2 Sinkowitz and Gottlieb, quoted by C. F. Coombs, Med. Annual, 198.
A8soc., July 31st, 1926. 